Review Article

\title{
The Role of von Willebrand Factor in Vascular Inflammation: From Pathogenesis to Targeted Therapy
}

\author{
Felice Gragnano, Simona Sperlongano, Enrica Golia, Francesco Natale, \\ Renatomaria Bianchi, Mario Crisci, Fabio Fimiani, Ivana Pariggiano, Vincenzo Diana, \\ Andreina Carbone, Arturo Cesaro, Claudia Concilio, Giuseppe Limongelli, \\ Mariagiovanna Russo, and Paolo Calabrò
}

\begin{abstract}
Division of Cardiology, Department of Cardio-Thoracic and Respiratory Sciences, University of Campania "Luigi Vanvitelli" and A.O. dei Colli Monaldi Hospital, Naples, Italy
\end{abstract}

Correspondence should be addressed to Paolo Calabrò; paolo.calabro@unicampania.it

Received 13 January 2017; Revised 15 March 2017; Accepted 23 March 2017; Published 28 May 2017

Academic Editor: Shengbo Cao

Copyright $\odot 2017$ Felice Gragnano et al. This is an open access article distributed under the Creative Commons Attribution License, which permits unrestricted use, distribution, and reproduction in any medium, provided the original work is properly cited.

\begin{abstract}
Beyond its role in hemostasis, von Willebrand factor (VWF) is an emerging mediator of vascular inflammation. Recent studies highlight the involvement of VWF and its regulator, ADAMTS13, in mechanisms that underlie vascular inflammation and immunothrombosis, like leukocyte rolling, adhesion, and extravasation; vascular permeability; ischemia/reperfusion injury; complements activation; and NETosis. The VWF/ADAMTS13 axis is implicated in the pathogenesis of atherosclerosis, promoting plaque formation and inflammation through macrophage and neutrophil recruitment in inflamed lesions. Moreover, VWF and ADAMTS13 have been recently proposed as prognostic biomarkers in cardiovascular, metabolic, and inflammatory diseases, such as diabetes, stroke, myocardial infarction, and sepsis. All these features make VWF an attractive therapeutic target in thromboinflammation. Several lines of research have recently investigated "tailor-made" inhibitors of VWF. Results from animal models and clinical studies support the potent anti-inflammatory and antithrombotic effect of VWF antagonism, providing reassuring data on its safety profile. This review describes the role of VWF in vascular inflammation "from bench to bedside" and provides an updated overview of the drugs that can directly interfere with the VWF/ADAMTS13 axis.
\end{abstract}

\section{Historical Perspective}

The story of von Willebrand factor starts in Finland in the first years of the 1900s. Professor Erik Adolf von Willebrand was an internist at the University of Helsinki interested in genetic and blood coagulation, and his studies led him to uncover a "new form of hemophilia", von Willebrand disease (VWD), the most common inherited bleeding disorder [1]. In 1925, he firstly examined Hjiordis, a 7-year-old girl from Föglö (Aland archipelago, Finland) who suffered from frequent and remarkable episodes of bleeding from the nose and lips following tooth extraction. He immediately noticed that joint bleeding, common in hemophilia, was rare. At the age of 13 years, Hjiordis died for a fatal bleeding during her fourth menstrual period. von Willebrand also mapped the family pedigree: both of her parents came from families with bleeding disorders, and all but two of her 12 siblings had bleeding symptoms (4 of them experienced fatal bleeding) [2]. In the '70s, several studies showed how factor VIII, impaired in hemophilia A, was not responsible for this hereditary disease. A newly discovered protein was recognized as the cause of hemorrhagic diathesis in these patients: the von Willebrand factor (VWF) $[3,4]$.

\section{VWF Architecture}

VWF is a large plasma adhesive glycoprotein with multimeric structure [5], selectively produced in megakaryocytes (MKs) and endothelial cells (ECs). Encoded on the short arm of chromosome 12, the basic monomer of VWF consists of 2.050 residues and contains four repeated domains assembled in the following order: D1-D2-D'-D3-A1-A2-A3-D4- 
C1-C2-C3-C4-C5-C6-CK [6]. A1 and A3 domains are mainly involved in thrombosis: A1 binds to GpIb $\alpha$ on the platelet surface and microfibrillar collagen (type VI); A3 binds to fibrillar collagens (types I and III) [7]. VWF multimerization is a multistep process: monomers of VWF firstly dimerize in the endoplasmic reticulum (ER); then, they link in mature VWF multimers or "concatemers" in the Golgi and post-Golgi compartments [7, 8]. Mature multimers of VWF are packaged in helicoidally structures and stored in Weibel-Palade bodies (WPBs) in ECs and $\alpha$-granules in MKs and platelets [7]. VWF molecules are produced and secreted with different sizes, ranging from dimers to ultralarge (UL) multimers (up to 100 monomers). In ECs, the release of VWF from WPBs occurs in both constitutive and regulated manner. On the other hand, platelet activation is necessary for VWF secretion from $\alpha$-granules and no constitutive release has been reported [9]. Ultralarge (UL) multimers of VWF (UL-VWF) are extremely reactive and hyperadhesive, prone to interact with platelets causing spontaneous platelet adhesion/aggregation. To avoid the accumulation of UL-VWF multimers, protein size is finely regulated by a metalloprotease, ADAMTS13 (a disintegrin and metalloprotease with thrombospondin motif). Under flow condition, ADAMTS13 binds to the A2 domain and, acting as enzymatic scissors, cleaves VWF "long-chain" multimers, and releases smaller, less active VWF molecules. The last tangle up in a globular quiescent conformation, hiding the A1 and A2 domains, involved, respectively, in VWF interaction with platelet and ADAMTS13 [10].

\section{Pathophysiology of VWF in the Bloodstream}

In intact vessels, at low-shear conditions, VWF circulates in a globular shape, as a "folded spring". However, VWF is highly dynamic in the bloodstream, and at high shear rates (above a threshold of $5000 \mathrm{~s}^{-1}$ ), globular-shaped VWF rapidly unfolds and elongates into a long-chain conformation, changing its functional status from inactive to highly reactive [11]. VWF interacts with platelets via two receptors: glycoprotein (Gp) Ib-IX-V complex and integrin $\alpha \mathrm{IIb} \beta 3$ [12]. In injured vessels, the VWF-GpIb interaction enables platelets to roll on damaged ECs and subendothelium and to establish a preliminary, unstable adhesive interaction. This low-affinity interaction gives time for the activation of the $\alpha \operatorname{IIb} \beta 3$ integrin that in turn binds to VWF, fibrinogen, fibrin, and different ECM (extracellular matrix) proteins leading to a more firm platelet adhesion and aggregation [7]. The role of VWF in platelet adhesion and aggregation is crucial under high-shear conditions (i.e., arterioles, microcirculation, and critical artery stenosis): at increasing shear rates (above $1000 \mathrm{~s}^{-1}$ ), aggregate formation is progressively more VWF-dependent. As a result, at very high shear rates (above $10,000 \mathrm{~s}^{-1}$ ), thrombus formation is almost exclusively mediated by the VWF-GpIb interaction [12]. VWF also participates indirectly in the coagulation cascade binding to factor VIII (FVIII). This interaction protects FVIII from proteolytic clearance prolonging its half-life and also improves its function directing its localization at the site of vascular injury [13]. Plasma level of VWF is determined by both genetic $[14,15]$ and nongenetic factors, such as $\mathrm{ABO}$ blood group, aging, and sexual hormones (i.e., estrogens). Endothelial dysfunction and activation, vascular aging, and arterial stiffness are all associated with increased levels of VWF $[16,17]$. Nitric oxide (NO), a marker of endothelial health, exerts an inhibitory effect on VWF release by ECs, probably blocking the granule membrane fusion process or inhibiting calcium mobilization from WPBs $[15,18,19]$. Endothelial dysfunction probably represents the background that links VWF, inflammation, and thrombosis [18-20].

\section{VWF in Vascular Inflammation}

The VWF/ADAMTS13 axis exerts a pivotal role in vascular inflammation and thrombosis [21]. Thrombosis, with the recruitment of platelets to the site of vessel's injury, and immune response, with the recruitment of leukocytes in inflamed tissues, have traditionally been considered two distinct pathways. This was the "dominant scientific view" until the new concept of "immunothrombosis" was introduced [22]. Several studies suggest how thrombosis can be considered a mechanism of intravascular immunity, limiting bacteria from spreading in the bloodstream. On the other hand, systemic inflammation and uncontrolled immunity response (i.e., sepsis) can lead to the extreme "pathological" activation of thrombotic cascade and disseminated intravascular coagulation (DIC) [22]. VWF may represent a possible "connection bridge" between the hemostatic and the inflammatory pathway (Figures 1 and 2) mediating adhesion and recruitment of both platelets and leukocytes: the network of cell interactions and aggregation is becoming more intriguing $[22,23]$.

4.1. VWF Secretion in Inflammation. Like C-reactive protein [24-27], VWF is an acute phase reactant [28], and its level increases in inflammatory and metabolic disorders (i.e., glucose intolerance, diabetes, and obesity) $[29,30]$. In contrast, similar to negative acute phase proteins, ADAMTS13 activity declines in patients with systemic inflammation [31]. As a result, inflammation may potentially activate thrombosis, inducing a marked imbalance between VWF and ADAMTS13. Different mediators of inflammation (cytokines, superoxide anions, histamine, and thrombin) produce an increase in VWF levels through various mechanisms $[32,33]$; for example, IL-8 and TNF- $\alpha$ significantly stimulate the release of UL-VWF by ECs, whereas IL-6 inhibits the UL-VWF cleavage by ADAMTS13. These data suggest that cytokines may potentially affect VWF metabolism, and in inflammatory disease, active UL-VWF multimers may accumulate in plasma and induce a prothrombotic state. However, this fascinating hypothesis needs confirmatory studies.

4.2. VWF and Leukocyte Recruitment: Rolling, Adhesion, and Extravasation. Leukocyte rolling, adhesion, and extravasation are hallmarks of inflammation [23, 34]. Initial rolling on endothelium results in a slowdown of circulating leukocytes, predominantly mediated by the interaction of selectins (P-selectin and E-selectin) on ECs and P-selectin 


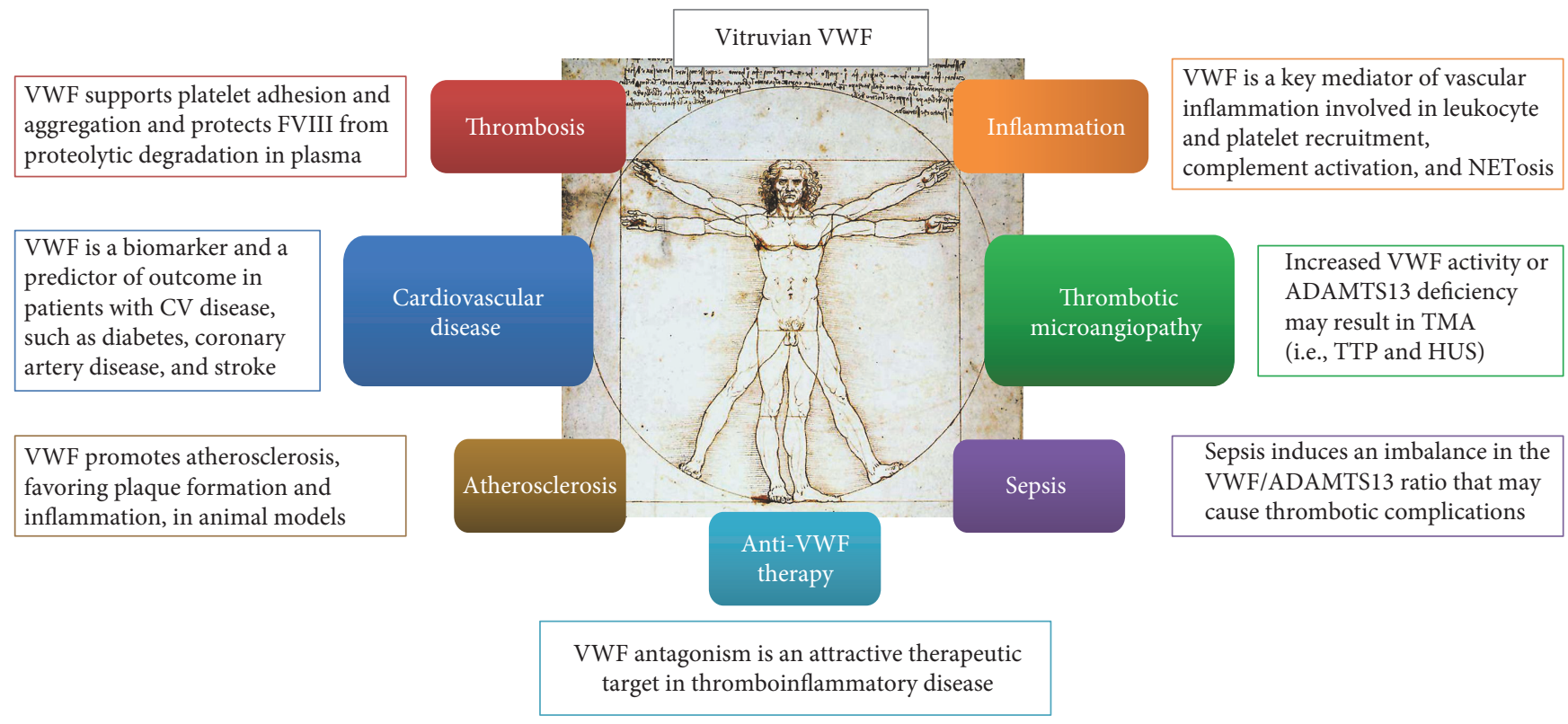

FIGURE 1: Functional heterogeneity of von Willebrand factor (VWF). VWF is best known for its role in hemostasis and thrombosis, supporting platelet adhesion/aggregation and protecting FVIII from proteolytic degradation in blood flow. However, it is now clear that VWF functions extend much further than that. VWF plays a key role in vascular inflammation, favoring leukocyte recruitment and extravasation, activating complement cascade, and participating in NETosis. In cardiovascular disease, including CAD and stroke, VWF is a predictor of future CV events. In atherosclerosis, VWF promotes plaque formation and inflammation in animal models. An increase in VWF activity or ADAMTS13 deficiency may result in microvascular obstruction and thrombotic microangiopathy (TMA). In sepsis, inflammatory and infective stimuli may induce an acute imbalance in the VWF/ADAMTS13 ratio with possible thrombotic complications (i.e., DIC). Finally, a growing interest is emerging on selective VWF antagonism as a new therapeutic option to provide a further advance in the treatment of thrombotic and inflammatory disorders. VWF: von Willebrand factor; ADAMTS13: a disintegrin and metalloprotease with the thrombospondin motif; TMA: thrombotic microangiopathy; CV: cardiovascular; CAD: coronary artery disease; TTP: thrombotic thrombocytopenic purpura; HUS: hemolytic uremic syndrome.

The solar system of VWF in inflammation

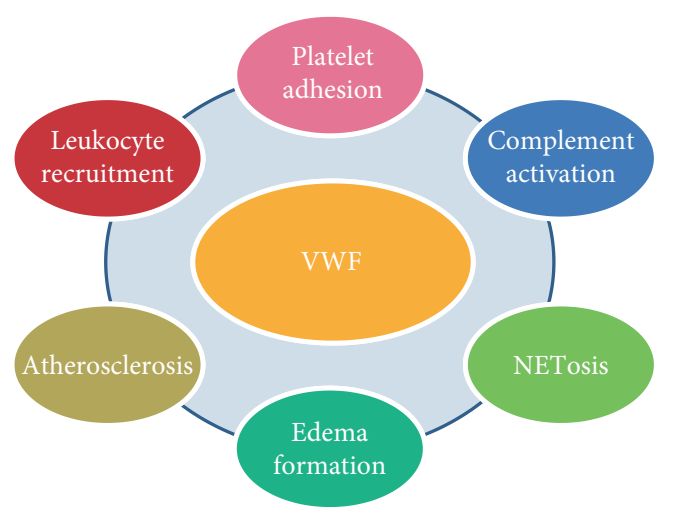

Figure 2: The "solar system" of VWF-mediated vascular inflammation. VWF is central in the "solar system" of vascular inflammation, and many inflammatory pathways orbit in its "gravitational field." VWF supports leukocyte and platelet recruitment in inflamed tissue, modulates vascular permeability and edema formation, may promote atherosclerotic plaque formation and inflammation, and provides an activating surface for complement activation and NETosis. All these mechanisms may contribute to tissue injury and organ failure in thromboinflammatory disorders. glycoprotein ligand-1 (PSGL-1) on leukocytes [35]. This unstable bond promotes leukocyte activation and stable adhesion on EC layer mediated by the interaction between leukocyte $\beta 2$-integrins $(\alpha \mathrm{L} \beta 2, \alpha \mathrm{M} \beta 2, \alpha \mathrm{D} \beta 2$, and $\alpha \mathrm{X} \beta 2)$ and endothelial intercellular adhesion molecules (ICAMs). This more stable interaction finally allows leukocyte transendothelial migration and extravasation [36]. In both venous and arterial environment (at low and high shear rates, respectively), leukocyte adhesion and extravasation may be supported by the VWF-leukocyte interaction (Figure 3).

4.2.1. VWF-Leukocyte Interaction in "Venous" Low-Shear Condition. In the ' 90 s, initial studies demonstrated a relation between VWF and leukocyte recruitment at a low shear rate. In 1993, Wagner firstly reported that VWF indirectly regulates the expression of P-selectin on the EC surface [37]. In 2001, Denis et al. confirmed these results in models of inflamed venules and, most importantly, showed how the absence of VWF correlates with a deficient leukocyte recruitment at a low shear rate $\left(95-100 \mathrm{~s}^{-1}\right)$ [38]. In the same year, Koivunen et al. showed that leukocytes could bind to VWF through the interaction between the leukocyte-specific $\beta 2$ integrins and the leucine-leucine-glycine (LLG) sequences contained in the A2 and D3 domains of VWF [39]. Some years later, Pendu et al. [23] clearly demonstrated that VWF could interact directly with polymorphonuclear leukocytes (PMNs), under static- and low-shear conditions (at wall 


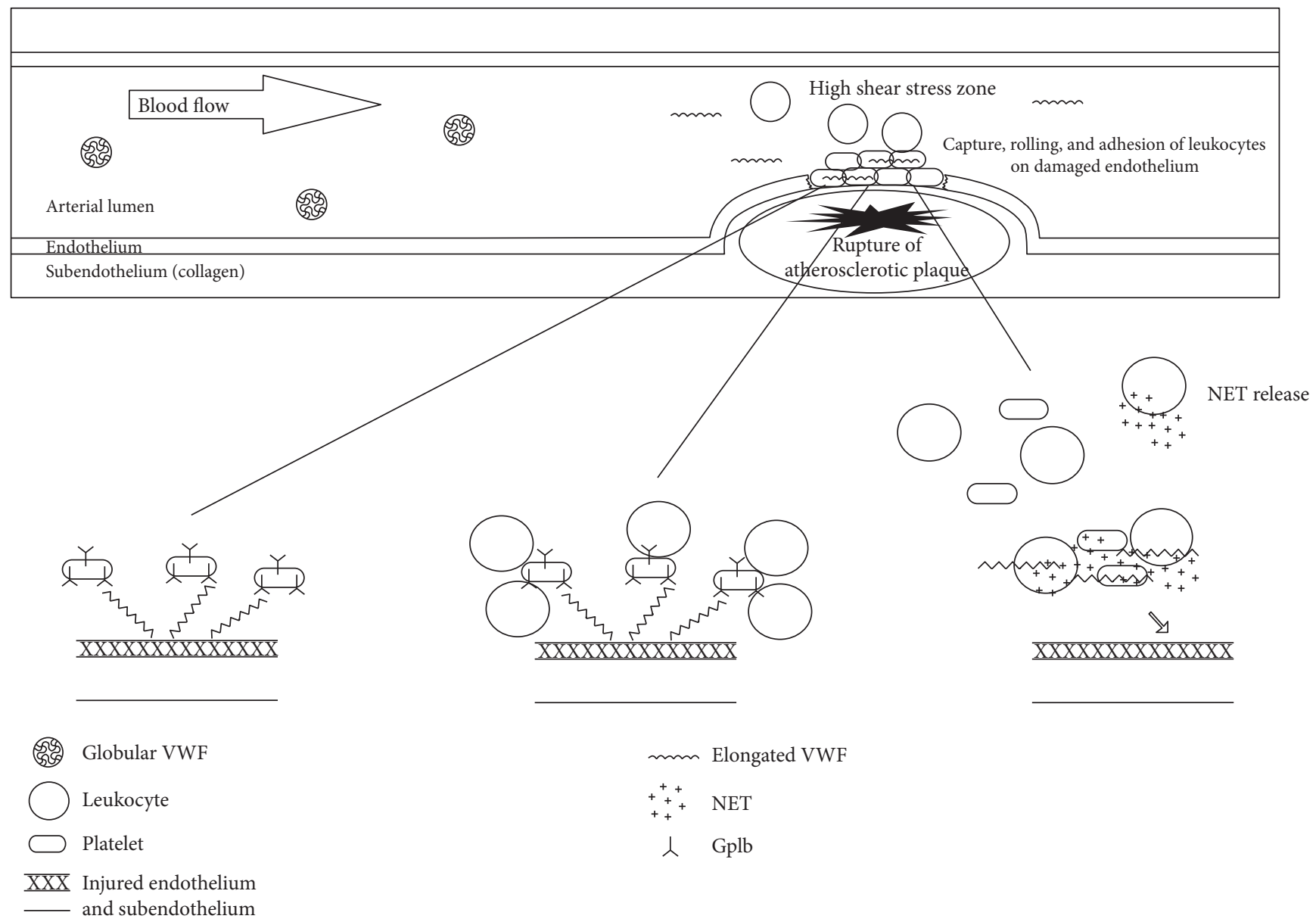

(a) VWF-platelet interaction on injured endothelium (b) Leukocytes anchored to VWF-platelet strings on injured endothelium (c) VWF and NETosis

FIGURE 3: VWF-mediated thromboinflammation in high-shear conditions. At high shear rates (e.g., arterioles, microcirculation, and artery stenosis), the inactive globular-shaped VWF rapidly unfolds and elongates in a highly reactive long-chain conformation. The elongated VWF can bind to platelets (a), allowing them to roll and adhere to the damaged endothelial surface. Platelet-decorated UL-VWF strings on activated endothelium represent a solid anchoring matrix for leukocyte adhesion (b), so permitting leukocyte recruitment in the inflamed site. In the bloodstream, VWF also binds to "neutrophil extracellular traps" (NETs) (c), inflammatory mediators (decondensed nucleosomes, extracellular DNA, and proteins) released by activated neutrophils, creating a network able to recruit both platelets and leukocytes and to promote thrombus formation.

shear rate: $50 \mathrm{~s}^{-1}$ ). All these results suggest that VWF may create an adhesive surface on activated ECs to capture monocytes and PMNs and mediate their stable (through $\beta 2$-integrins) and unstable (through PSGL-1) adhesion [23, 39].

\subsubsection{VWF-Leukocyte Interaction in "Arterial" High-Shear} Condition. The "adhesive" mechanisms described above, efficient at a low shear rate, probably change under high-shear stress conditions (i.e., arterial and arteriolar flows) where a more complex interaction among VWF, leukocytes, and platelets is necessary. Bernardo et al. [40] showed how, under high-shear stress (20-40 dynes $\left./ \mathrm{cm}^{2}\right)$, leukocytes tethered and rolled on an ideal matrix of platelets adherent to UL-VWF strings on activated ECs, but not directly on the endothelial layer. In this model, platelet-decorated UL-VWF strings represent a firm anchor for leukocyte adhesion. Authors speculate that this mechanism may support the accumulation of leukocytes in inflamed vascular lesions, such as arterial atherosclerotic plaques.

4.2.3. VWF and Leukocyte Extravasation. Beyond rolling and adhesion, VWF may also contribute to the extravasation of leukocytes in inflamed tissues. In a murine model of thioglycollate-induced peritonitis, Petri et al. [41] showed how VWF mediates PMN extravasation, modulating endothelial barrier permeability. Also, in their model intravenous injection of anti-VWF or anti-GpIb antibodies inhibited PMN recruitment across the inflamed endothelium, demonstrating that VWF-mediated PMN extravasation is strictly dependent on the presence of platelets and GpIb. Looking at their results, the authors suggest that in the inflamed vessel, platelet interaction with VWF anchored on activated ECs could promote the opening of endothelial junctions and thereby facilitates the leukocyte diapedesis process. 
4.3. VWF and Complement System. The complement system is an enzyme cascade with a defensive role against infection, diffusely involved in the pathogenesis of inflammatory and thrombotic disorders (i.e., autoimmune diseases, ischemiareperfusion injury, hemolytic uremic syndrome [HUS], and thrombocytopenic purpura [TTP]) [42]. Three complement pathways have been described: classical, alternative, and lectin. They all converge into a final common generation of the membrane attack complex (MAC), the cytotoxic component of the complement system [42]. Recent evidence suggests that VWF may promote the activation of alternative complement pathway (AP). In experimental models of the human umbilical vein ECs (HUVECs) [43], complement products have been detected to be attached on VWF strings. VWF multimers anchored on ECs create an "activating surface" for AP: complement compounds may bind to the ECanchored VWF strings and promote cascade activation [43] leading to the final formation of the MAC. In thrombotic microangiopathies (TMA) (i.e., TTP and HUS), ADAMTS13 deficiency has been associated with abnormal complement activation [44]. As known, many patients experienced TTP and HUS episodes during inflammatory conditions (i.e., infections) [45]. In these view, both ADAMTS13 deficiency and inflammatory stimuli may increase the concentration of UL-VWF strings on ECs and, in turn, trigger AP activation, with an uncontrolled synthesis of anaphylatoxins/ MAC that aggravates tissue injury $[43,44,46]$.

4.4. VWF, NETosis, and HNPs: Thrombosis and Host Defense. Neutrophils are principal actors in immunothrombosis [47] and have two points of contact with the VWF system: human neutrophil peptides (HNPs) and NETosis. HNPs, also known as $\alpha$-defensins, are small cationic antimicrobial peptides released from activated neutrophils, involved as mediators of vascular inflammation in metabolic and inflammatory disorders (including acute coronary syndrome [ACS]) [48]. In 2016, Pillai et al. [49] demonstrated an inhibitory effect of HPNs on ADAMTS13 proteolytic activity and an increased level of HPNs in patients with acquired TTP. NETosis is a recently discovered cell death process that links sterile inflammation and thrombosis [50]. In inflammatory condition, activated neutrophils release "neutrophil extracellular traps" (NETs): inflammatory mediators with a cytotoxic effect, formed by decondensed nucleosomes, extracellular DNA, and proteins derived from intracellular granules, such as neutrophil elastase (NE) and myeloperoxidase (MPO) [51]. VWF can bind to NETs [52], and the VWF-NET network may mediate both leukocyte and platelet recruitment in high-shear conditions (Figure 3). Interestingly, heparin may interfere with DNA-VWF binding and blocks leukocyte adhesion. In this view, the VWF-NET interaction could promote vascular inflammation and may be a potential target for combined anti-inflammatory and antithrombotic therapies [52-54].

\section{VWF in Atherogenesis}

The role of VWF in atherogenesis is still unclear. In the ECs of the atherosclerotic lesion, there is a high concentration of
WPBs (in which the VWF in stored) [55] and oxidized LDLs and high-shear stress, two factors involved in atherosclerosis, can induce WPB exocytosis [56, 57]. In the '70s, Fuster et al. observed that homozygous VWF-deficient (VWF -/-) pigs hardly developed aortic atherosclerosis whereas $\mathrm{VWF}+/+$ pigs showed significant atherosclerosis [58]. In 2001, Methia et al. demonstrated that early atherosclerotic lesions, fatty streak and early fibrous plaques, formed in the aortic sinus of VWF $-/-$ mice were smaller and contained fewer macrophages than those in $\mathrm{VWF}+/+$ mice. Moreover, in VWF $+/+$ mice, atherosclerotic lesions were mostly located at the branch points of the arteries (known as regions of disturbed flow), whereas they were not so prominent in these areas in mice with VWF deficiency [59]. In 2003, Qin et al. proved in vitro that VWF directly stimulates the proliferation of smooth muscular cells (SMCs), one of the major constituents of atherosclerotic plaques, in a direct dose-dependent way and that low-shear stress in vivo accelerates intimal hyperplasia proportional to VWF expression [60]. Also, ADAMTS13 indirectly modulates atherosclerosis by cleaving UL-VWF multimers that may actively participate in the macrophage and neutrophil recruitment in inflamed plaques, with a potential protective effect on atherosclerotic lesion progression [61]. Finally, it has been observed in animal models that anti-VWF agents have a protective effect against atherogenesis [62]. Molecules that disturb the interaction between VWF and GpIb, like monoclonal antibodies AJvW-2 [62] and AJW200 [63], the VWF recombinant fragment VCL [64], and ATA (aurintricarboxylic acid) [65] have shown to prevent neointima formation and growth, by inhibiting platelet adhesion to the vessel wall and SMC proliferation. The link between VWF and atherogenesis has been recently enriched by recent studies on the interaction between high-density lipoprotein (HDL), apolipoproteinAI (ApoA-I), and VWF. As a principal actor in reverse cholesterol transport (RCT), HDL has antiatherogenic proprieties. Chung et al. recently demonstrated how HDL/ ApoA-I can exert an anti-VWF effect reducing VWF secretion, preventing self-association of hyperactive UL-VWF multimers, and interfering with the capacity of VWF to bind to the vessel wall [66]. Although this evidence is in animal models, an unequivocal protective role of VWF deficiency in atherosclerosis has not been demonstrated in humans. Indeed, autopsy studies showed that even patients with congenital VWD type 3 (complete VWF deficiency) were not fully protected from atherosclerosis [67]. Therefore, additional studies are needed to clarify VWF's real role in atherosclerosis.

\section{VWF in Metabolic and Cardiovascular Disease: Animal Models and Clinical Evidence}

6.1. Animal Models. The role of VWF in stroke has been diffusely investigated in animal models. In mouse models of ischemic stroke, VWF activates thromboinflammatory pathways involved in postischemic inflammatory response and ischemia/reperfusion (I/R) injury, and complete 
deficiency of VWF is protective for cerebral infarction $[68,69]$. Conversely, mice lacking ADAMTS13 have enhanced inflammatory response following I/R brain injury, increased infarct volume, and more severe neurological deficits compared with wild-type (WT) mice $[68,70]$. ADAMTS13 -/mice [70] also exhibited enhanced neutrophil infiltration in the infarcted and peri-infarcted region and increased activity of the inflammatory markers (such as MPO, TNF- $\alpha$, and IL-6). This enhanced acute inflammatory response in mice lacking ADAMTS13 has been shown to be entirely mediated by VWF [70]. Similarly, several studies on mice suggest an involvement of the VWF/ADAMTS13 axis in myocardial ischemia/reperfusion injury [71, 72]. Finally, VWF could aggravate $I / R$ injury through its capacity of promoting complement activation $[73,74]$.

6.2. Clinical Studies. VWF and ADAMTS13 have been proposed as useful biomarkers and predictors of prognosis in patients with cardiovascular and metabolic disease [75]. In diabetic patients, VWF and ADAMTS13 activities correlate with $\mathrm{CV}$ outcome and risk of chronic complications and may predict a response to therapies $[30,76]$. In the Rotterdam study, elevated VWF levels predict the highest risk of ischemic stroke in general population [77]. Conversely, patients with VWD have shown a reduced risk of ischemic stroke [78]. Furthermore, in patients with chronic cerebrovascular disease, VWF levels are higher than those in healthy individuals, but lower than those in acute ischaemic stroke/ TIA patients, suggesting a crescent gradient of VWF activity among normal, chronic, and acute cerebrovascular conditions [79]. In this view, VWF may become a hopeful target in stroke management [21]. Recent evidence also suggests a clinical relation between VWF and coronary artery disease. In the European Concerted Action on Thrombosis and Disabilities (ECAT) study [80], in stable patients with angiographically documented coronary artery disease (CAD), higher levels of VWF:antigen (VWF:Ag) were independently associated with an increased incidence of MI and sudden death. More recently, in the ATHEROREMO-IVUS study [81], in patients with stable CAD (SCAD), an increased level of VWF:Ag was associated with higher coronary plaque burden, adverse CV outcome, and death during 1-year of follow-up. VWF is also an independent risk factor for first STEMI: levels of VWF are significantly increased in patients with first ST-elevation myocardial infarction (STEMI) rather than in controls [82]. Finally, Marcucci et al. reported a possible relation between lower levels of ADAMTS13 and higher residual platelet reactivity (RPR), a marker of resistance to antiplatelet therapy associated with increased risk of ischemic events [83]. These results confirm a cross-talk between thrombosis and inflammation suggesting that the VWF and ADAMTS13 may influence both clinical outcome and response to therapy in metabolic and cardiovascular disease.

\section{VWF, TMA, and Infective Disease: Cross-Talk between Infection and Thrombosis}

Dysfunctional activation of the VWF/ADAMTS13 system in thrombotic microangiopathies (TMAs) (such as TTP, HUS, and DIC) results in microvessel occlusion by a complex web of VWF-rich thrombi causing tissue hypoperfusion and organ failure, potentially life-threatening [84]. As known, TMAs are possible complications of infective disease and sepsis [45]. In infective disease, the presence of inflammatory stimuli may trigger an acute imbalance in the VWF/ADAMTS13 system through an increase in VWF multimer secretion and an inhibition of ADAMTS13 activity [85] and consequently lead to microvascular occlusion and multiorgan failure. In this view, it is interesting to observe how in clinical studies on patients with SIRS and sepsis, VWF and ADAMTS13 have shown to be a prognostic biomarker identifying patients with worse outcome and a higher risk of death [86-88]. The ADAMTS13 pattern also differed in infective (sepsis) or noninfective SIRS [89]: septic patients have lower levels of ADAMTS13 than patients with noninfectious SIRS. These studies together bring new lights on the link between thrombosis, inflammation, and infective diseases. Further clinical studies are needed to clarify if restoration of normal activity levels of ADAMTS13 (i.e., with plasma or recombinant ADAMTS13 infusions) may neutralize VWF-system prevalence and prevent microvascular occlusion and organ failure in patients with SIRS and sepsis.

\section{VWF in Vasculitis}

A recent study in a model of vascular inflammation provides food for thoughts in VWF research. Hillgruber et al. [90] described the impact of VWF on in leukocytoclastic vasculitis (LcV), an immune complex- (IC-) mediated vasculitis (ICV) common in dermatology, caused by the precipitation of IC in the vessel wall and subsequent recruitment of neutrophils. Compared with those of healthy skin controls, a massive accumulation of VWF was present in skin biopsies of patients suffering from $\mathrm{LcV}$. This result was also confirmed in experimental murine models of ICV, identifying ICs as potent VWF secretagogues in ECs. Also, VWF promoted leukocyte recruitment and edema formation, probably regulating endothelial permeability $[90,91]$. To test antiinflammatory properties of anti-VWF therapy, Hillgruber et al. used polyclonal anti-VWF antibodies able to block epitopes in the D'-D3 region (that mediate the VWF/leukocyte interaction) without interfering with the GpIba-VWF interaction. Interestingly, anti-VWF treatment showed a vascular anti-inflammatory effect blocking leukocyte recruitment and edema formation and was useful in both prophylactic and therapeutic administrations. Of note, no interference with hemostasis was registered. This result confirms VWF antagonism as a promising target therapy in inflammatory vascular disorders.

\section{Anti-VWF Target Therapy in Thromboinflammation}

Targeting the VWF/ADMTS13 pathway in thromboinflammation with specific drugs is a fascinating hypothesis (Table 1). 
TABLE 1: VWF-targeted pharmacotherapy. Specific and nonspecific VWF antagonists and mechanism of action. VWF: von Willebrand factor; GpIb: glycoprotein Ib; Fab: fragment antigen binding; TNF: tumor necrosis factor; MA: monoclonal antibody; Ref.: references.

\begin{tabular}{|c|c|c|}
\hline Drugs & Mechanism of action & Ref. \\
\hline \multicolumn{3}{|l|}{ Nonspecific VWF antagonists } \\
\hline Statins & Anti-inflammatory effect (?) & {$[94]$} \\
\hline Heparin & Binds VWF-A1 domain + anti-inflammatory effect (?) & [95-97] \\
\hline N-Acetylcysteine (NAC) & ADAMTS13-like activity & [98-100] \\
\hline Anti-TNF $\alpha$ & Anti-inflammatory effect (?) & {$[93]$} \\
\hline Corticosteroids & Anti-inflammatory effect (?) & {$[92]$} \\
\hline Colchicine & Microtubule disruption & {$[32]$} \\
\hline Aurintricarboxylic acid (ATA) & Inhibits VWF-GpIb interaction & {$[65]$} \\
\hline \multicolumn{3}{|l|}{ Specific VWF antagonists } \\
\hline h6B4-Fab & MA: inhibits VWF-GpIb interaction & [103] \\
\hline GPG-290 & MA: inhibits VWF-GpIb interaction & [104] \\
\hline AJvW-2 & MA: inhibits VWF-GpIb interaction & {$[62]$} \\
\hline AJW200 & MA: inhibits VWF-GpIb interaction & {$[102,105$} \\
\hline 82D6A3 & MA: inhibits VWF-collagen interaction & {$[107]$} \\
\hline SZ-123 & MA: inhibits VWF interaction with GpIb and collagen & {$[109]$} \\
\hline ARC1779 & Aptamers: inhibits VWF-GpIb binding & {$[111-113$} \\
\hline ARC15105 & Aptamers: inhibits VWF-collagen binding & {$[114]$} \\
\hline ALX-0081 (caplacizumab) & Nanobody: inhibits VWF-GpIb interaction & {$[117]$} \\
\hline VCL & VWF recombinant fragment & {$[64]$} \\
\hline BAX930 & Recombinant ADAMTS13 (rADAMTS13) & [123] \\
\hline
\end{tabular}

9.1. Nonspecific Anti-VWF Therapy. Daily-used drugs have demonstrated a VWF-modulating activity in different clinical conditions. Anti-inflammatory agents such as corticosteroids [92] or TNF $\alpha$ inhibitors [93] block the release of acute phase reactants, including VWF. Colchicine, an alkaloid with anti-inflammatory effects, inhibits VWF release inducing microtubule disruption [32]. Statin, lipid-lowering medications with anti-inflammatory effects, recently demonstrated to significantly reduce plasma levels of VWF [94]. Lowmolecular weight heparins (LMWHs) can, directly and indirectly, antagonize VWF activity through antithrombotic and anti-inflammatory mechanisms [95-97]. N-Acetylcysteine (NAC) is an important antioxidant with anti-inflammatory properties [98]. Recent findings highlighted the ability of NAC to exert a direct negative modulation of VWF, mimicking ADAMTS13 activity, degrading UL-VWF multimers, and inhibiting VWF cell interaction (with platelets and leukocytes) by the disruption of the disulfide bond in the VWF A1 domain [99]. Several studies also suggest the benefit of NAC in patients with severe TTP crisis [100] probably blocking both proinflammatory and prothrombotic effects of VWF. In summary, unselective VWF antagonism of daily-used drugs may play beneficial in inflammatory and thrombotic disorders. However, data are not sufficient, and the beneficial effect of the anti-VWF action is hard to quantify in terms of benefit/risk ratio. To clarify the potential benefit of VWF antagonism, specific drugs need to be tested in preclinical and clinical studies.

9.2. Specific Anti-VWF Therapy. Specific VWF antagonism represents a new interesting issue in thromboinflammation therapy with a potential role in metabolic and cardiovascular disease. Leukocyte adhesion and extravasation, vascular permeability, edema formation, abnormal complement activation, ischemia-reperfusion injury, NETosis, and inflammatory-induced microvascular thrombosis are all potential therapeutic targets. Experimental models suggest that the neutralization of VWF activity using specific pharmacological compounds could result in both antithrombotic and anti-inflammatory effects $[71,101]$. Recently, different classes of drugs (including antibodies, nanobodies, and aptamers) have been tested in preclinical and clinical studies. Further studies are needed for clinical approval.

9.2.1. Antibodies. Anti-VWF antibodies have been widely tested in animal models. Pendu et al. demonstrated how the use of specific antibodies directed to the D'D3 region permits to selectively block VWF function in vascular inflammation, without interfering with its hemostatic function [23]. Hillgruber et al. [90] used polyclonal VWF-directed antibodies to target the D'D3 region in models of cutaneous inflammation inducing an immediate regression of inflammatory response and a significant reduction in leukocyte recruitment and vascular permeability. Also in this case, the antiinflammatory effect did not interfere with hemostasis [90]. Monoclonal antibodies have also been tested in animal models of cardiovascular disease (coronary thrombosis, stroke, and in-stent stenosis): GPG-290, 6B4-Fab, h6B4Fab, and AJW200 directly target the VWF-GpIb binding; 82D6A3 blocks VWF-collagen interaction; and finally, SZ-123 inhibits both mechanisms [102-109]. All compounds demonstrated a powerful antithrombotic effect 
and possible anti-inflammatory proprieties [102] with no important side effects, in terms of spontaneous bleeding and thrombocytopenia [102-109].

9.2.2. Aptamers. The aptamer is a new pharmacological class composed of small RNA/DNA molecules, of 20 to 100 nucleotides, highly specific and nonimmunogenic [110]. Two molecules have been tested in preclinical and clinical studies: ARC1779 that targets VWF A1 domain-GpIb interaction [111-113] and ARC15105 that targets A1 domain on VWF and blocks VWF-collagen binding [114, 115]. Both showed an effective inhibition of VWF activity with a significant antiplatelet effect, without causing serious adverse events [111-115], but no anti-inflammatory effect has been reported.

9.2.3. Nanobodies. Nanobodies represent a novel class of highly specific therapeutic proteins with broad application prospects in research and clinical practice [116]. ALX-0081 (caplacizumab) is an anti-VWF humanized nanobody that selectively targets the A1 domain, blocking the VWF-GpIb interaction. In the TITAN trial [117], a randomized placebo-controlled phase 2 study, 75 patients with acquired TTP were randomly assigned to subcutaneous caplacizumab (10 mg daily) or placebo, as an adjunct to standard therapy. Caplacizumab induced a more rapid resolution of the acute TTP episode and more efficient organ protection, associated with an increased tendency toward mild/moderate bleeding as compared with the placebo group [117].

9.2.4. Recombinant ADAMTS13 Therapy. Recombinant ADAMTS13 (rADAMTS13) therapy showed antiinflammatory and antithrombotic effects [118] on preclinical models of cerebrovascular disease and TTP [119-123]. In mouse models of stroke treated with tissue plasminogen activator (tPA) $[118,120]$, rADAMTS13 was protective against ischemic brain injury, prevented inflammation-induced cerebral endothelial damage [119], and reduced tPA-associated hemorrhage, probably by regulating the blood-brain barrier (BBB) integrity [120]. These results confirm previous findings [68] reporting that infusion of rADAMTS13 reduces infarct volume and improves ischemic stroke outcome without producing cerebral hemorrhage. In mouse models of intracerebral hemorrhage (ICH) [121], treatment with rADAMTS13 reduced chemokine and cytokine level, adhesion molecule (ICAM-1) expression, metalloprotease (MMP-9) and MPO activity, and microglia activation. The protective effect of $\mathrm{rADAMTS} 13$ also determined a reduction in brain edema and neutrophil recruitment, with a better preservation of the $\mathrm{BBB}$ integrity as compared with that of the control group. Interestingly, on cultured ECs, the antiinflammatory effect of rADAMTS13 was reversed by recombinant von Willebrand factor (rVWF), suggesting that VWF mediates the effect of ADAMTS13 on vascular inflammation. Finally, in ADAMTS13 -/- mouse models of TTP, the use of a new rADAMTS13 product (BAX930) [123] demonstrated both prophylactic and therapeutic efficacies, with a favorable preclinical profile, supporting future clinical development. Looking at recent studies, specific anti-
VWF and recombinant ADAMTS13 therapies represent an appealing field of research. Initial results are encouraging, even if no definitive clinical data are available.

\section{Conclusions}

VWF is a key mediator of vascular inflammation. Recent lines of evidence suggest its role in leukocyte and platelet recruitment in inflamed tissue. In experimental models, VWF supports the activation of multiple inflammatory pathways, such as complement cascade and NETosis, promotes atherosclerosis favoring plaque progression and complication, and exacerbates ischemia/reperfusion injury. In patients with cardiovascular disease, including CAD and stroke, VWF and ADAMTS13 are both predictors of future CV events. All these findings suggest that selective VWF antagonism is an attractive therapeutic option to provide further advances in the treatment of thrombotic and inflammatory vascular disorders. In the next future, more preclinical and clinical studies are expected to throw open new avenues of investigation into the VWF/ADAMTS13 system.

\section{Abbreviations \\ Fab: $\quad$ Fragment antigen binding \\ STEMI: ST-elevation myocardial infarction \\ TTP: $\quad$ Thrombotic thrombocytopenic purpura \\ UL-VWF: Ultralarge von Willebrand factor \\ VWF: von Willebrand factor \\ VWF:Ag: von Willebrand factor antigen.}

\section{Conflicts of Interest}

The authors declared no potential conflicts of interest with respect to the research, authorship, and/or publication of this article.

\section{Authors' Contributions}

All authors contributed to (1) the conception and design of the article, (2) drafting the article or revising it critically for important intellectual content, and (3) the final approval of the version to be published.

\section{Acknowledgments}

This work was supported by a grant from the Italian Ministry for Education, University and Research (FIRB RBFR12W5V5), to the corresponding authors.

\section{References}

[1] E. A. Willebrand, "Hereditar pseudohemofili," Finska Lakarsallskapets Hand, vol. 1, no. 67, pp. 7-112, 1926.

[2] I. M. Nilsson, "In memory of Erik Jorpes. von Willebrand's disease from 1926-1983," Scandinavian Journal of Haematology. Supplementum, vol. 40, no. 33, pp. 21-43, 1984.

[3] D. Meyer and M. J. Larrieu, "Von Willebrand factor and platelet adhesiveness," Journal of Clinical Pathology, vol. 23, no. 3, pp. 228-231, 1970. 
[4] T. S. Zimmerman, O. D. Ratnoff, and A. E. Powell, "Immunologic differentiation of classic hemophilia (factor VIII deficiency) and von Willebrand's disease, with observations on combined deficiencies of antihemophilic factor and proaccelerin (factor V) and on an acquired circulating anticoagulant against antihemophilic factor," The Journal of Clinical Investigation, vol. 50, no. 1, pp. 244-254, 1971.

[5] P. J. Lenting, C. Casari, O. D. Christophe, and C. V. Denis, "von Willebrand factor: the old, the new and the unknown," Journal of Thrombosis and Haemostasis, vol. 10, no. 12, pp. 2428-2437, 2012.

[6] Y. F. Zhou, E. T. Eng, J. Zhu, C. Lu, T. Walz, and T. A. Springer, "Sequence and structure relationships within von Willebrand factor," Blood, vol. 120, no. 2, pp. 449-458, 2012.

[7] M. Bryckaert, J. P. Rosa, C. V. Denis, and P. J. Lenting, "Of von Willebrand factor and platelets," Cellular and Molecular Life Sciences, vol. 72, no. 2, pp. 307-326, 2015.

[8] T. A. Springer, "von Willebrand factor, Jedi knight of the bloodstream," Blood, vol. 124, no. 9, pp. 1412-1425, 2014.

[9] A. Padilla, J. L. Moake, A. Bernardo et al., "P-selectin anchors newly released ultralarge von Willebrand factor multimers to the endothelial cell surface," Blood, vol. 103, no. 6, pp. 2150-6, 2004.

[10] J. T. Crawley, R. de Groot, Y. Xiang, B. M. Luken, and D. A. Lane, "Unraveling the scissile bond: how ADAMTS13 recognizes and cleaves von Willebrand factor," Blood, vol. 118, no. 12, pp. 3212-3221, 2011.

[11] S. Sorrentino, J. D. Studt, O. Medalia, and K. Tanuj Sapra, "Roll, adhere, spread and contract: structural mechanics of platelet function," European Journal of Cell Biology, vol. 94, no. 3-4, pp. 129-138, 2015.

[12] S. P. Jackson, "The growing complexity of platelet aggregation," Blood, vol. 109, no. 12, pp. 5087-5095, 2007.

[13] Z. M. Ruggeri, "Structure of von Willebrand factor and its function in platelet adhesion and thrombus formation," Best Practice \& Research. Clinical Haematology, vol. 14, no. 2, pp. 257-279, 2001.

[14] N. L. Smith, M. H. Chen, A. Dehghan et al., "Novel associations of multiple genetic loci with plasma levels of factor VII, factor VIII, and von Willebrand factor: The CHARGE (Cohorts for Heart and Aging Research in Genome Epidemiology) Consortium," Circulation, vol. 121, no. 12, pp. 1382-1392, 2010.

[15] U. M. Vischer, "von Willebrand factor, endothelial dysfunction, and cardiovascular disease," Journal of Thrombosis and Haemostasis, vol. 4, no. 6, pp. 1186-1193, 2006.

[16] U. M. Vischer, F. R. Herrmann, T. Peyrard, R. Nzietchueng, and A. Benetos, "Plasma von Willebrand factor and arterial aging," Journal of Thrombosis and Haemostasis, vol. 3, no. 4, pp. 794-795, 2005.

[17] J. A. van Mourik, R. Boertjes, I. A. Huisveld et al., "von Willebrand factor propeptide in vascular disorders: a tool to distinguish between acute and chronic endothelial cell perturbation," Blood, vol. 94, no. 1, pp. 179-185, 1999.

[18] D. A. Brott, A. Katein, H. Thomas et al.., "Evaluation of von Willebrand factor and von Willebrand factor propeptide in models of vascular endothelial cell activation, perturbation, and/or injury," Toxicologic Pathology, vol. 42, no. 4, pp. 672-683, 2014.

[19] S. Zarei, M. Frieden, J. E. Kaufmann, and U. M. Vischer, "The regulation of endothelial VWF secretion by nitric oxide: is it physiological?" Journal of Thrombosis and Haemostasis, vol. 4, no. 1, pp. 263-265, 2006.

[20] M. Schwameis, C. Schörgenhofer, A. Assinger, M. M. Steiner, and B. Jilma, "VWF excess and ADAMTS13 deficiency: a unifying pathomechanism linking inflammation to thrombosis in DIC, malaria, and TTP," Thrombosis and Haemostasis, vol. 113, no. 4, pp. 708-718, 2015.

[21] S. F. De Meyer, F. Denorme, F. Langhauser, E. Geuss, F. Fluri, and C. Kleinschnitz, "Thromboinflammation in stroke brain damage," Stroke, vol. 47, no. 4, pp. 1165-1172, 2016.

[22] B. Engelmann and S. Massberg, "Thrombosis as an intravascular effector of innate immunity," Nature Reviews. Immunology, vol. 13, no. 1, pp. 34-45, 2013.

[23] R. Pendu, V. Terraube, O. D. Christophe et al., "P-selectin glycoprotein ligand 1 and beta2-integrins cooperate in the adhesion of leukocytes to von Willebrand factor," Blood, vol. 108, no. 12, pp. 3746-3752, 2006.

[24] E. Golia, G. Limongelli, F. Natale et al., "Adipose tissue and vascular inflammation in coronary artery disease," World Journal of Cardiology, vol. 6, no. 7, pp. 539-554, 2014.

[25] L. Forte, G. Cimmino, F. Loffredo et al., "C-reactive protein is released in the coronary circulation and causes endothelial dysfunction in patients with acute coronary syndromes," International Journal of Cardiology, vol. 152, no. 1, pp. 7-12, 2011.

[26] P. Calabrò, E. Golia, and E. T. Yeh, "Role of C-reactive protein in acute myocardial infarction and stroke: possible therapeutic approaches," Current Pharmaceutical Biotechnology, vol. 13, no. 1, pp. 4-16, 2012.

[27] B. Memoli, A. Procino, P. Calabrò et al., "Inflammation may modulate IL-6 and C-reactive protein gene expression in the adipose tissue: the role of IL-6 cell membrane receptor," American Journal of Physiology. Endocrinology and Metabolism, vol. 293, no. 4, pp. E1030-E1035, 2007.

[28] B. E. Pottinger, R. C. Read, E. M. Paleolog, P. G. Higgins, and J. D. Pearson, "von Willebrand factor is an acute phase reactant in man," Thrombosis Research, vol. 53, no. 4, pp. 387-394, 1989.

[29] J. B. Meigs, M. A. Mittleman, D. M. Nathan et al., "Hyperinsulinemia, hyperglycemia, and impaired hemostasis: the Framingham Offspring Study," Jama, vol. 283, no. 2, pp. 221-228, 2000.

[30] A. Jager, V. W. van Hinsbergh, P. J. Kostense et al., "von Willebrand factor, C-reactive protein, and 5-year mortality in diabetic and nondiabetic subjects: the Hoorn Study," Arteriosclerosis, Thrombosis, and Vascular Biology, vol. 19, no. 12, pp. 3071-3078, 1999.

[31] C. L. Bockmeyer, R. A. Claus, U. Budde et al., "Inflammationassociated ADAMTS13 deficiency promotes formation of ultra-large von Willebrand factor," Haematologica, vol. 93, no. 1, pp. 137-140, 2008.

[32] U. M. Vischer, H. Barth, and C. B. Wollheim, "Regulated von Willebrand factor secretion is associated with agonist-specific patterns of cytoskeletal remodeling in cultured endothelial cells," Arteriosclerosis, Thrombosis, and Vascular Biology, vol. 20, no. 3, pp. 883-891, 2000.

[33] A. Bernardo, C. Ball, L. Nolasco, J. F. Moake, and J. F. Dong, "Effects of inflammatory cytokines on the release and cleavage of the endothelial cell-derived ultralarge von Willebrand factor multimers under flow," Blood, vol. 104, no. 1, pp. 100-106, 2004. 
[34] K. Ley, "Molecular mechanisms of leukocyte recruitment in the inflammatory process," Cardiovascular Research, vol. 32, no. 4, pp. 733-742, 1996.

[35] R. P. McEver and R. D. Cummings, "Perspectives series: cell adhesion in vascular biology: role of PSGL-1 binding to selectins in leukocyte recruitment," The Journal of Clinical Investigation, vol. 100, no. 3, pp. 485-491, 1997.

[36] C. Cerletti, V. Evangelista, and G. de Gaetano, "P-selectinbeta 2-integrin cross-talk: a molecular mechanism for polymorphonuclear leukocyte recruitment at the site of vascular damage," Thrombosis and Haemostasis, vol. 82, no. 2, pp. 787-793, 1999.

[37] D. D. Wagner, "The Weibel-Palade body: the storage granule for von Willebrand factor and P-selectin," Thrombosis and Haemostasis, vol. 70, no. 1, pp. 105-110, 1993.

[38] C. V. Denis, P. André, S. Saffaripour, and D. D. Wagner, "Defect in regulated secretion of P-selectin affects leukocyte recruitment in von Willebrand factor-deficient mice," Proceedings of the National Academy of Sciences of the United States of America, vol. 98, no. 7, pp. 4072-4077, 2001.

[39] E. Koivunen, T. M. Ranta, A. Annila et al., "Inhibition of $\beta 2$ integrin-mediated leukocyte cell adhesion by leucine-leucine-glycine motif-containing peptides," The Journal of Cell Biology, vol. 153, no. 5, pp. 905-916, 2001.

[40] A. Bernardo, C. Ball, L. Nolasco, H. Choi, J. L. Moake, and J. F. Dong, "Platelets adhered to endothelial cellbound ultra-large von Willebrand factor strings support leukocyte tethering and rolling under high shear stress," Journal of Thrombosis and Haemostasis, vol. 3, no. 3, pp. 562-570, 2005.

[41] B. Petri, A. Broermann, H. Li et al., "von Willebrand factor promotes leukocyte extravasation," Blood, vol. 116, no. 22, pp. 4712-4719, 2010.

[42] J. M. Thurman and V. M. Holers, "The central role of the alternative complement pathway in human disease," Journal of Immunology, vol. 176, no. 3, pp. 1305-1310, 2006.

[43] N. A. Turner and J. Moake, "Assembly and activation of alternative complement components on endothelial cell-anchored ultra-large von Willebrand factor links complement and hemostasis-thrombosis," PloS One, vol. 8, no. 3, article e59372, 2013.

[44] N. Turner, L. Nolasco, J. Nolasco, S. Sartain, and J. Moake, "Thrombotic microangiopathies and the linkage between von Willebrand factor and the alternative complement pathway," Seminars in Thrombosis and Hemostasis, vol. 40, no. 5, pp. 544-550, 2014.

[45] C. M. Cserti, S. Landaw, and L. Uhl, "Do infections provoke exacerbations and relapses of thrombotic thrombocytopenic purpura?" Journal of Clinical Apheresis, vol. 22, no. 1, pp. 21-25, 2007.

[46] M. Réti, P. Farkas, D. Csuka et al., "Complement activation in thrombotic thrombocytopenic purpura," Journal of Thrombosis and Haemostasis, vol. 10, no. 5, pp. 791-798, 2012.

[47] K. Kambas, I. Mitroulis, and K. Ritis, “The emerging role of neutrophils in thrombosis-the journey of TF through NETs," Frontiers in Immunology, vol. 3, p. 385, 2012.

[48] K. Quinn, M. Henriques, T. Parker, A. S. Slutsky, and H. Zhang, "Human neutrophil peptides: a novel potential mediator of inflammatory cardiovascular diseases," American Journal of Physiology. Heart and Circulatory Physiology, vol. 295, no. 5, pp. H1817-H1824, 2008.
[49] V. G. Pillai, J. Bao, C. B. Zander et al., "Human neutrophil peptides inhibit cleavage of von Willebrand factor by ADAMTS13: a potential link of inflammation to TTP," Blood, vol. 128, no. 1, pp. 110-119, 2016.

[50] K. Martinod and D. D. Wagner, "Thrombosis: tangled up in NETs," Blood, vol. 123, no. 18, pp. 2768-2776, 2014.

[51] T. A. Fuchs, A. Brill, D. Duerschmied et al., "Extracellular DNA traps promote thrombosis," Proceedings of the National Academy of Sciences of the United States of America, vol. 107, no. 36, pp. 15880-15885, 2010.

[52] J. Riegger, R. A. Byrne, M. Joner et al., "Histopathological evaluation of thrombus in patients presenting with stent thrombosis. A multicenter European study: a report of the prevention of late stent thrombosis by an interdisciplinary global European effort consortium," European Heart Journal, vol. 37, no. 19, pp. 1538-1549, 2016.

[53] S. Grässle, V. Huck, K. I. Pappelbaum et al., "von Willebrand factor directly interacts with DNA from neutrophil extracellular traps," Arteriosclerosis, Thrombosis, and Vascular Biology, vol. 34, no. 7, pp. 1382-1389, 2014.

[54] R. Oklu, H. Albadawi, M. T. Watkins, M. Monestier, M. Sillesen, and S. Wicky, "Detection of extracellular genomic DNA scaffold in human thrombus: implications for the use of deoxyribonuclease enzymes in thrombolysis," Journal of Vascular and Interventional Radiology, vol. 23, no. 5, pp. 712-718, 2012.

[55] A. A. Trillo and R. W. Prichard, "Early endothelial changes in experimental primate atherosclerosis," Laboratory Investigation, vol. 41, no. 4, pp. 294-302, 1979.

[56] D. K. Vora, Z. T. Fang, S. M. Liva et al., "Induction of Pselectin by oxidized lipoproteins. Separate effects on synthesis and surface expression," Circulation Research, vol. 80, no. 6, pp. 810-818, 1997.

[57] M. Galbusera, C. Zoja, R. Donadelli et al., "Fluid shear stress modulates von Willebrand factor release from human vascular endothelium," Blood, vol. 90, no. 4, pp. 1558-1564, 1997.

[58] V. Fuster, E. J. W. Bowie, J. C. Lewis, D. N. Fass, C. A. Owen Jr., and A. L. Brown, "Resistance to arteriosclerosis in pigs with von Willebrand's disease," The Journal of Clinical Investigation, vol. 61, no. 3, pp. 722-730, 1978.

[59] N. Methia, P. André, C. V. Denis, M. Economopoulos, and D. D. Wagner, "Localized reduction of atherosclerosis in von Willebrand factor-deficient mice," Blood, vol. 98, no. 5, pp. 1424-1428, 2001.

[60] F. Qin, T. Impeduglia, P. Schaffer, and H. Dardik, "Overexpression of von Willebrand factor is an independent risk factor for pathogenesis of intimal hyperplasia: preliminary studies," Journal of Vascular Surgery, vol. 37, no. 2, pp. 433-439, 2003.

[61] C. Gandhi, A. Ahmad, K. M. Wilson, and A. K. Chauhan, "ADAMTS13 modulates atherosclerotic plaque progression in mice via a VWF-dependent mechanism," Journal of Thrombosis and Haemostasis, vol. 12, no. 2, pp. 255-260, 2014.

[62] S. Kageyama, H. Yamamoto, and R. Yoshimoto, "Antihuman von Willebrand factor monoclonal antibody AJvW2 prevents thrombus deposition and neointima formation after balloon injury in guinea pigs," Arteriosclerosis, Thrombosis, and Vascular Biology, vol. 20, no. 10, pp. 2303-2308, 2000. 
[63] A. Yamashita, Y. Asada, H. Sugimura et al., "Contribution of von Willebrand factor to thrombus formation on neointima of rabbit stenotic iliac artery under high blood-flow velocity," Arteriosclerosis, Thrombosis, and Vascular Biology, vol. 23, no. 6, pp. 1105-1110, 2003.

[64] D. Zahger, M. C. Fishbein, L. I. Garfinkel et al., "VCL, an antagonist of the platelet GP1b receptor, markedly inhibits platelet adhesion and intimal thickening after balloon injury in the rat," Circulation, vol. 92, no. 5, pp. 1269-1273, 1995.

[65] H. Matsuno, O. Kozawa, M. Niwa, and T. Uematsu, "Inhibition of von Willebrand factor binding to platelet GP Ib by a fractionated aurintricarboxylic acid prevents restenosis after vascular injury in hamster carotid artery," Circulation, vol. 96, no. 4, pp. 1299-1304, 1997.

[66] D. W. Chung, J. Chen, M. Ling et al., "High-density lipoprotein modulates thrombosis by preventing von Willebrand factor self-association and subsequent platelet adhesion," Blood, vol. 127, no. 5, pp. 637-645, 2016.

[67] A. B. Federici, P. M. Mannucci, E. Fogato, P. Ghidoni, and L. Matturri, "Autopsy findings in three patients with von Willebrand disease type IIB and type III: presence of atherosclerotic lesions without occlusive arterial thrombi," Thrombosis and Haemostasis, vol. 70, no. 5, pp. 758-761, 1993.

[68] B. Q. Zhao, A. K. Chauhan, M. Canault et al., "von Willebrand factor-cleaving protease ADAMTS13 reduces ischemic brain injury in experimental stroke," Blood, vol. 114, no. 15, pp. 3329-3334, 2009.

[69] C. Kleinschnitz, S. F. De Meyer, T. Schwarz et al., "Deficiency of von Willebrand factor protects mice from ischemic stroke," Blood, vol. 113, no. 15, pp. 3600-3603, 2009.

[70] M. M. Khan, D. G. Motto, S. R. Lentz, and A. K. Chauhan, "ADAMTS13 reduces VWF-mediated acute inflammation following focal cerebral ischemia in mice," Journal of Thrombosis and Haemostasis, vol. 10, no. 8, pp. 1665-1671, 2012.

[71] C. Gandhi, D. G. Motto, M. Jensen, S. R. Lentz, and A. K. Chauhan, "ADAMTS13 deficiency exacerbates VWFdependent acute myocardial ischemia/reperfusion injury in mice," Blood, vol. 120, no. 26, pp. 5224-5230, 2012.

[72] S. F. De Meyer, A. S. Savchenko, M. S. Haas et al., "Protective anti-inflammatory effect of ADAMTS13 on myocardial ischemia/reperfusion injury in mice," Blood, vol. 120, no. 26, pp. 5217-5223, 2012.

[73] A. Elvington, C. Atkinson, H. Zhu et al., "The alternative complement pathway propagates inflammation and injury in murine ischemic stroke," Journal of Immunology, vol. 189, no. 9, pp. 4640-4647, 2012.

[74] J. Mocco, W. J. Mack, A. F. Ducruet et al., "Complement component C3 mediates inflammatory injury following focal cerebral ischemia," Circulation Research, vol. 99, no. 2, pp. 209-217, 2006.

[75] F. Gragnano, E. Golia, F. Natale et al., "The role of von Willebrand factor in cardiovascular disease: from a biochemical marker to an attractive therapeutic target," Current Vascular Pharmacology, vol. 15, pp. 1-12, 2017.

[76] E. Rurali, M. Noris, A. Chianca, and et al.BENEDICT Study Group, "ADAMTS13 predicts renal and cardiovascular events in type 2 diabetic patients and response to therapy," Diabetes, vol. 62, no. 10, pp. 3599-3609, 2013.

[77] R. G. Wieberdink, M. C. van Schie, P. J. Koudstaal et al., "High von Willebrand factor levels increase the risk of stroke: the Rotterdam study," Stroke, vol. 41, no. 10, pp. 2151-2156, 2010.

[78] Y. V. Sanders, J. Eikenboom, E. M. de Wee et al., "Reduced prevalence of arterial thrombosis in von Willebrand disease," Journal of Thrombosis and Haemostasis, vol. 11, no. 5, pp. 845-854, 2013.

[79] P. Kraft, C. Drechsler, I. Gunreben et al., "Von Willebrand factor regulation in patients with acute and chronic cerebrovascular disease: a pilot, case-control study," PloS One, vol. 9, no. 6 article e99851, 2014.

[80] S. G. Thompson, J. Kienast, S. D. Pyke, F. Haverkate, and J. C. van de Loo, "Hemostatic factors and the risk of myocardial infarction or sudden death in patients with angina pectoris. European Concerted Action on Thrombosis and Disabilities Angina Pectoris Study Group," The New England Journal of Medicine, vol. 332, no. 10, pp. 635-641, 1995.

[81] M. A. Sonneveld, J. M. Cheng, R. M. Oemrawsingh et al., "Von Willebrand factor in relation to coronary plaque characteristics and cardiovascular outcome. Results of the ATHEROREMO-IVUS study," Thrombosis and Haemostasis, vol. 113, no. 3, pp. 577-584, 2015.

[82] B. Rutten, A. Maseri, D. Cianflone et al., "Plasma levels of active von Willebrand factor are increased in patients with first ST-segment elevation myocardial infarction: a multicenter and multiethnic study," European Heart Journal. Acute Cardiovascular Care, vol. 4, no. 1, pp. 64-74, 2015.

[83] R. Marcucci, F. Cesari, S. Cinotti et al., "ADAMTS-13 activity in the presence of elevated von Willebrand factor levels as a novel mechanism of residual platelet reactivity in high risk coronary patients on antiplatelet treatment," Thrombosis Research, vol. 123, no. 1, pp. 130-136, 2008.

[84] S. R. Cataland and H. M. Wu, "Acquired thrombotic thrombocytopenic purpura: new therapeutic options and their optimal use," Journal of Thrombosis and Haemostasis, vol. 13, Supplement 1, pp. S223-S229, 2015.

[85] T. Ono, J. Mimuro, S. Madoiwa et al., "Severe secondary deficiency of von Willebrand factor-cleaving protease (ADAMTS13) in patients with sepsis-induced disseminated intravascular coagulation: its correlation with development of renal failure," Blood, vol. 107, no. 2, pp. 528-534, 2006.

[86] K. Martin, D. Borgel, N. Lerolle et al., "Decreased ADAMTS-13 (a disintegrin-like and metalloprotease with thrombospondin type 1 repeats) is associated with a poor prognosis in sepsis-induced organ failure," Critical Care Medicine, vol. 35, no. 10, pp. 2375-2382, 2007.

[87] V. Peigne, E. Azoulay, I. Coquet et al., "The prognostic value of ADAMTS13 (a disintegrin and metalloprotease with thrombospondin type 1 repeats, member 13) deficiency in septic shock patients involves interleukin-6 and is not dependent on disseminated intravascular coagulation," Critical Care, vol. 17, no. 6, p. R273, 2013.

[88] A. Hyseni, H. Kemperman, D. W. de Lange, J. Kesecioglu, P. G. de Groot, and M. Roest, "Active von Willebrand factor predicts 28-day mortality in patients with systemic inflammatory response syndrome," Blood, vol. 123, no. 14, pp. 2153-2156, 2014.

[89] J. Aibar, P. Castro, G. Espinosa et al., "ADAMTS-13 in critically ill patients with septic syndromes and noninfectious systemic inflammatory response syndrome," Shock, vol. 43, no. 6, pp. 556-562, 2015.

[90] C. Hillgruber, A. K. Steingräber, B. Pöppelmann et al., "Blocking von Willebrand factor for treatment of cutaneous 
inflammation," The Journal of Investigative Dermatology, vol. 134, no. 1, pp. 77-86, 2014.

[91] J. Huang, R. Roth, J. E. Heuser, and J. E. Sadler, "Integrin alpha(v)beta(3) on human endothelial cells binds von Willebrand factor strings under fluid shear stress," Blood, vol. 113, no. 7, pp. 1589-1597, 2009.

[92] M. D. de Kruif, L. C. Lemaire, I. A. Giebelen et al., "Prednisolone dose-dependently influences inflammation and coagulation during human endotoxemia," Journal of Immunology, vol. 178, no. 3, pp. 1845-1851, 2007.

[93] R. A. DeLa Cadena, A. Majluf-Cruz, A. Stadnicki et al., "Recombinant tumor necrosis factor receptor p75 fusion protein (TNFR:Fc) alters endotoxin-induced activation of the kinin, fibrinolytic, and coagulation systems in normal humans," Thrombosis and Haemostasis, vol. 80, no. 1, pp. 114-118, 1998.

[94] A. Sahebkar, C. Serban, S. Ursoniu et al., "The impact of statin therapy on plasma levels of von Willebrand factor antigen. Systematic review and meta-analysis of randomised placebo-controlled trials," Thrombosis and Haemostasis, vol. 115, no. 3, pp. 520-532, 2016.

[95] G. Montalescot, J. P. Collet, L. Lison et al., "Effects of various anticoagulant treatments on von Willebrand factor release in unstable angina," Journal of the American College of Cardiology, vol. 36, no. 1, pp. 110-114, 2000.

[96] S. Mousavi, M. Moradi, T. Khorshidahmad, and M. Motamedi, "Anti-inflammatory effects of heparin and its derivatives: a systematic review," Advances in Pharmacological Sciences, vol. 2015, p. 507151, 2015.

[97] G. Montalescot, C. Bal-dit-Sollier, D. Chibedi et al., "Comparison of effects on markers of blood cell activation of enoxaparin, dalteparin, and unfractionated heparin in patients with unstable angina pectoris or non-ST-segment elevation acute myocardial infarction (the ARMADA study)," The American Journal of Cardiology, vol. 91, no. 8, pp. 925-930, 2003.

[98] I. Elbini Dhouib, M. Jallouli, A. Annabi, N. Gharbi, S. Elfazaa, and M. M. Lasram, "A minireview on N-acetylcysteine: an old drug with new approaches," Life Sciences, vol. 151, pp. 359-363, 2016.

[99] J. Chen, A. Reheman, F. C. Gushiken et al., "N-Acetylcysteine reduces the size and activity of von Willebrand factor in human plasma and mice," The Journal of Clinical Investigation, vol. 121, no. 2, pp. 593-603, 2011.

[100] F. A. Sayani and C. S. Abrams, "How I treat refractory thrombotic thrombocytopenic purpura," Blood, vol. 125, no. 25, pp. 3860-3867, 2015.

[101] F. Denorme and S. F. De Meyer, "The VWF-GPIb axis in ischaemic stroke: lessons from animal models," Thrombosis and Haemostasis, vol. 116, no. 4, pp. 597-604, 2016.

[102] P. A. Lapchak, S. Doyan, X. Fan, and C. M. Woods, "Synergistic effect of AJW200, a von Willebrand factor neutralizing antibody with low dose $(0.9 \mathrm{mg} / \mathrm{mg})$ thrombolytic therapy following embolic stroke in rabbits," Journal of Neurology \& Neurophysiology, vol. 4, no. 2, p. 146, 2013.

[103] A. Fontayne, M. Meiring, S. Lamprecht et al., "The humanized anti-glycoprotein Ib monoclonal antibody h6B4-Fab is a potent and safe antithrombotic in a high shear arterial thrombosis model in baboons," Thrombosis and Haemostasis, vol. 100, no. 4, pp. 670-677, 2008.
[104] M. Wadanoli, D. Sako, G. D. Shaw et al., "The von Willebrand factor antagonist (GPG-290) prevents coronary thrombosis without prolongation of bleeding time," Thrombosis and Haemostasis, vol. 98, no. 2, pp. 397-405, 2007.

[105] S. Kageyama, H. Yamamoto, H. Nakazawa et al., "Pharmacokinetics and pharmacodynamics of AJW200, a humanized monoclonal antibody to von Willebrand factor, in monkeys," Arteriosclerosis, Thrombosis, and Vascular Biology, vol. 22, no. 1, pp. 187-192, 2002.

[106] H. Yamamoto, I. Vreys, J. M. Stassen, R. Yoshimoto, J. Vermylen, and M. F. Hoylaerts, "Antagonism of vWF inhibits both injury induced arterial and venous thrombosis in the hamster," Journal of Thrombosis and Haemostasis, vol. 79, no. 1, pp. 202-210, 1998.

[107] S. Staelens, M. A. Hadders, S. Vauterin et al., "Paratope determination of the antithrombotic antibody 82D6A3 based on the crystal structure of its complex with the von Willebrand factor A3-domain," The Journal of Biological Chemistry, vol. 281, no. 4, pp. 2225-2231, 2006.

[108] S. F. De Meyer, S. Staelens, P. N. Badenhorst et al., "Coronary artery in-stent stenosis persists despite inhibition of the von Willebrand factor-collagen interaction in baboons," Thrombosis and Haemostasis, vol. 98, no. 6, pp. 1343-1349, 2007.

[109] Y. M. Zhao, M. Jiang, S. D. Ji et al., "Anti-human VWF monoclonal antibody SZ-123 prevents arterial thrombus formation by inhibiting VWF-collagen and VWF-platelet interactions in Rhesus monkeys," Biochemical Pharmacology, vol. 85, no. 7, pp. 945-953, 2013.

[110] X. Ni, M. Castanares, A. Mukherjee, and S. E. Lupold, "Nucleic acid aptamers: clinical applications and promising new horizons," Current Medicinal Chemistry, vol. 18, no. 27, pp. 4206-4214, 2011.

[111] A. O. Spiel, F. B. Mayr, N. Ladani et al., "The aptamer ARC1779 is a potent and specific inhibitor of von Willebrand factor mediated ex vivo platelet function in acute myocardial infarction," Platelets, vol. 20, no. 5, pp. 334-340, 2009.

[112] H. S. Markus, C. McCollum, C. Imray, M. A. Goulder, J. Gilbert, and A. King, "The von Willebrand inhibitor ARC1779 reduces cerebral embolization after carotid endarterectomy: a randomized trial," Stroke, vol. 42, no. 8, pp. 2149-2153, 2011.

[113] P. Knöbl, B. Jilma, J. C. Gilbert, R. M. Hutabarat, P. G. Wagner, and P. Jilma-Stohlawetz, "Anti-von Willebrand factor aptamer ARC1779 for refractory thrombotic thrombocytopenic purpura," Transfusion, vol. 49, no. 10, pp. 2181-2185, 2009.

[114] J. M. Siller-Matula, Y. Merhi, J. F. Tanguay et al., “ARC15105 is a potent antagonist of von Willebrand factor mediated platelet activation and adhesion," Arteriosclerosis, Thrombosis, and Vascular Biology, vol. 32, no. 4, pp. 902-909, 2012.

[115] J. A. Diaz, S. K. Wrobleski, C. M. Alvarado et al., "P-selectin inhibition therapeutically promotes thrombus resolution and prevents vein wall fibrosis better than enoxaparin and an inhibitor to von Willebrand factor," Arteriosclerosis, Thrombosis, and Vascular Biology, vol. 35, no. 4, pp. 829$837,2015$.

[116] O. Y. Dmitriev, S. Lutsenko, and S. Muyldermans, "Nanobodies as probes for protein dynamics in vitro and in cells," The Journal of Biological Chemistry, vol. 291, no. 8, pp. 3767-3775, 2016. 
[117] F. Peyvandi, M. Scully, J. A. Kremer Hovinga et al., "Caplacizumab for acquired thrombotic thrombocytopenic purpura," The New England Journal of Medicine, vol. 374, no. 6, pp. 511-522, 2016.

[118] A. K. Chauhan, D. G. Motto, C. B. Lamb et al., "Systemic antithrombotic effects of ADAMTS13," The Journal of Experimental Medicine, vol. 203, no. 3, pp. 767-776, 2006.

[119] T. Nakano, K. Irie, K. Hayakawa et al., "Delayed treatment with ADAMTS13 ameliorates cerebral ischemic injury without hemorrhagic complication," Brain Research, vol. 1624, pp. 330-335, 2015.

[120] L. Wang, W. Fan, P. Cai et al., "Recombinant ADAMTS13 reduces tissue plasminogen activator-induced hemorrhage after stroke in mice," Annals of Neurology, vol. 73, no. 2, pp. 189-198, 2013.

[121] P. Cai, H. Luo, H. Xu et al., "Recombinant ADAMTS 13 attenuates brain injury after intracerebral hemorrhage," Stroke, vol. 46, no. 9, pp. 2647-2653, 2015.

[122] C. Muroi, M. Fujioka, K. Mishima et al., "Effect of ADAMTS13 on cerebrovascular microthrombosis and neuronal injury after experimental subarachnoid hemorrhage," Journal of Thrombosis and Haemostasis, vol. 12, no. 4, pp. 505-514, 2014.

[123] A. Kopić, K. Benamara, C. Piskernik et al., "Preclinical assessment of a new recombinant ADAMTS-13 drug product (BAX930) for the treatment of thrombotic thrombocytopenic purpura," Journal of Thrombosis and Haemostasis, vol. 14, no. 7, pp. 1410-1419, 2016. 


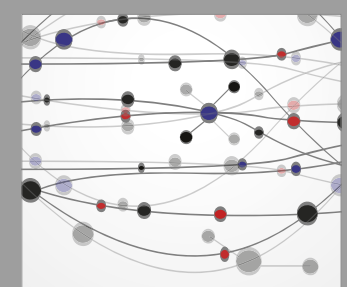

The Scientific World Journal
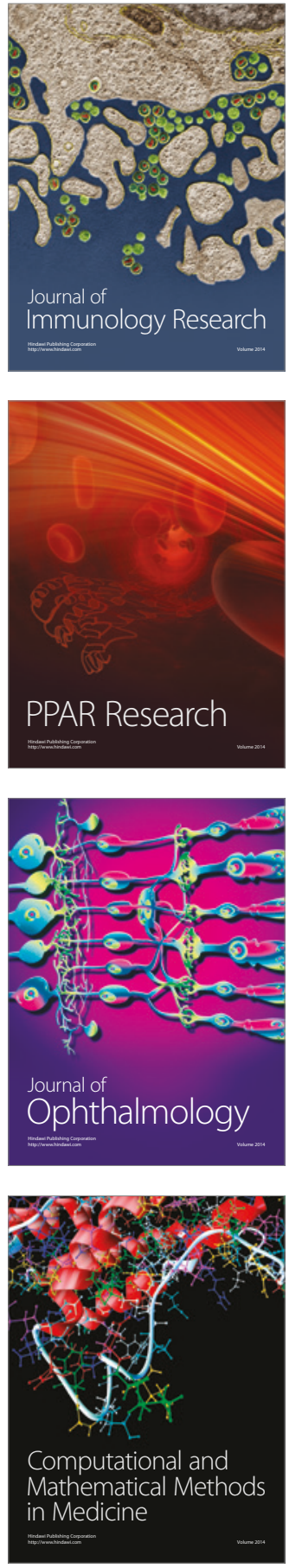

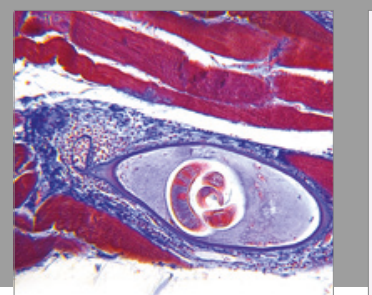

Gastroenterology Research and Practice
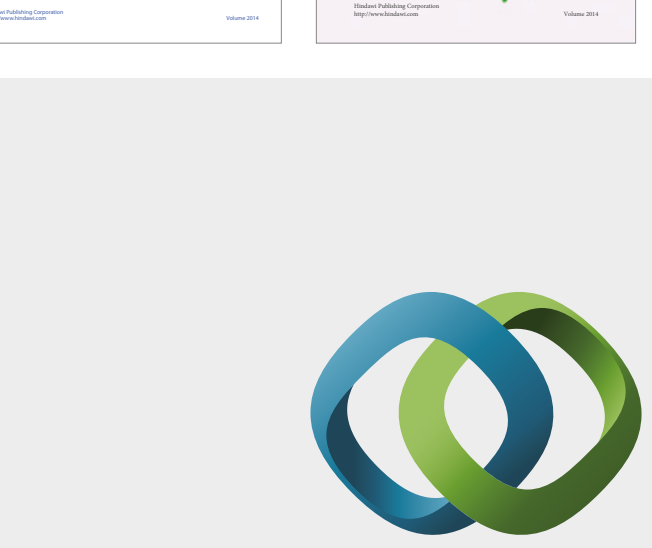

\section{Hindawi}

Submit your manuscripts at

https://www.hindawi.com
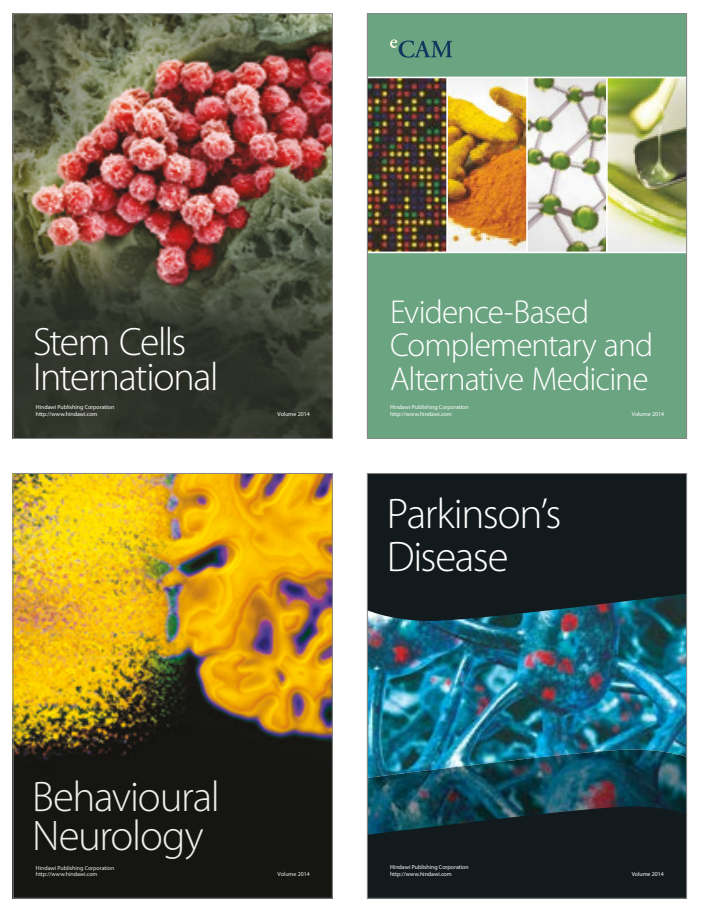
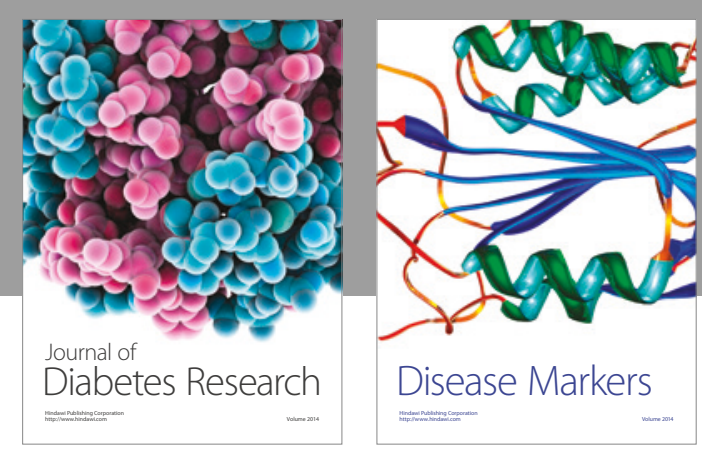

Disease Markers
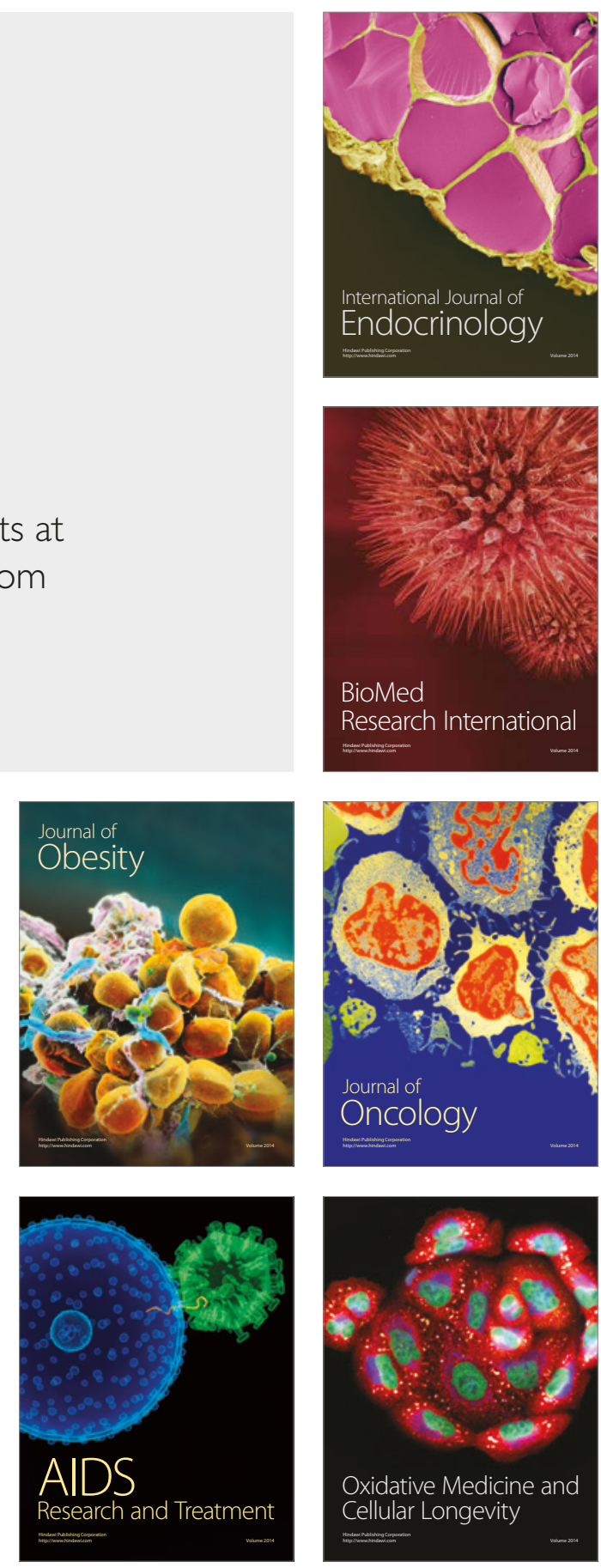